\title{
Separate modal analysis for tumor detection with a Digital Image Elasto Tomography (DIET) breast cancer screening system.
}

\author{
Adrianus M.W. Heeren ${ }^{\dagger}$, Thomas F. Lotz ${ }^{\ddagger}$, Amer Kashif ${ }^{\ddagger}$, J. Geoffrey Chase ${ }^{\ddagger}$ \\ $\dagger^{\dagger}$ Department of Mechanical Engineering, \\ Eindhoven University of Technology, \\ Eindhoven, The Netherlands \\ $\ddagger$ Centre for Bioengineering, Department of Mechanical Engineering, \\ University of Canterbury, \\ Christchurch, New Zealand
}

\begin{abstract}
Digital Image Elasto Tomography (DIET) is a non-invasive breast cancer screening modality that induces mechanical vibrations into a breast and images its surface motion with digital cameras. A new approach in software based diagnosis of this surface motion is presented, focussing on the second natural frequency of the breast. Separate modal analysis is used to estimate the modal parameters using imaging data from silicone phantoms. The second natural frequency proves to be a reliable metric with the potential to clearly distinguish cancerous and healthy tissue as well as providing an approximate location for the tumor. Furthermore, thorough statistical analysis is performed to verify the results.
\end{abstract}

\section{Introduction}

Breast cancer is a major public health issue all over the world, with over a million women estimated to be diagnosed with it every year, and more than 400,000 mortalities [1]. Statistics show that early detection and intervention greatly increases five year survival rates to over $95 \%$ [2, 3], stressing the need for effective wide-scale screening programmes.

X-ray mammography is currently the only approved large scale breast screening modality, being widely used in most developed countries. However, mammography raises controversy due to low compliance, poor sensitivity [4], accumulated radiation exposure [5], discomfort due to breast compression [6] and over-diagnosis [7]. Furthermore, breast cancer screening programs are often not available in developing countries due to relatively high costs involved [8] and rural populations with limited access to specialized radiology clinics [9], requiring a low cost and less invasive modality to better meet screening demands.

The Digital Image Elasto Tomography (DIET) system is a breast cancer screening approach to detect cancerous lesions based on a three dimensional representation of the elastic properties throughout the 


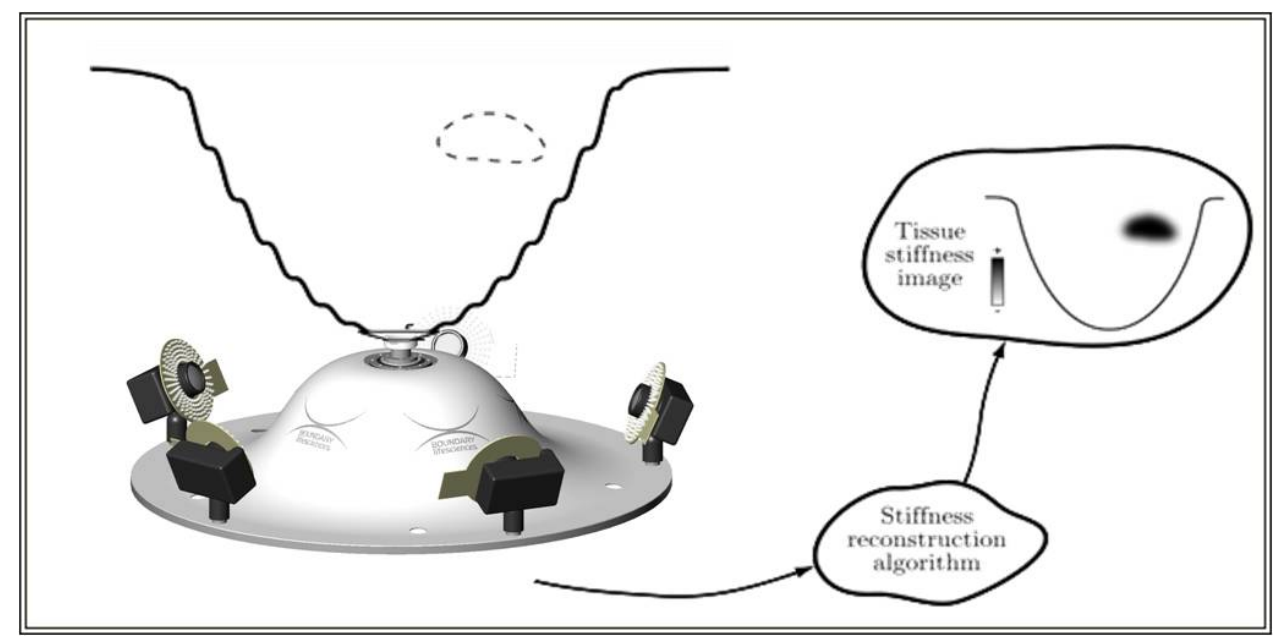

Figure 1: Schematic of the imaging principle in the DIET system.

breast volume. This technology is non-invasive, does not require breast compression, is very portable, requires low operator skill (software based diagnosis) and is low in operational cost [10, rendering this approach to be very suitable for large screening programs, particulary in remote areas or countries with limited access to screening.

The DIET system makes use of the high contrast difference in elastic properties of $300-1500 \%$ between cancerous tissue and healthy fatty or fibroglandular tissue in the breast [11, compared to a $5-10 \%$ radio-density contrast imaged in X-ray mammography [2].

In the DIET system mechanical sinusoidal vibrations are applied to the breast and the resulting surface oscillations are captured in three dimensions (3D) by an array of digital cameras surrounding the breast, tracking fiducial markers on the breast's surface [12, 13, 14, as shown in Figure 1. The surface vibration response is analyzed to detect disturbances in the observed vibration patterns, indicating a higher stiffness area within the tissue. This response is used to determine the probability and angular location of a potentially cancerous inclusion. Finite element [12] (FEM) or surface based methods [10, 15] can be used to provide an estimate of the location and the size of the inclusion. While FEM can provide a full 3D elastographic map of the breast, the inverse solutions involved are typically computationally expensive [16.

Surface based methods as in [10 show promising results and act as a proof of concept, but rely on a visual inspection of the surface response at different imaging frequencies. The minimal modeling method presented in [15] uses a broader range of imaging frequencies and fits a simple mechanical model to the frequency response of the breast surface, providing a more automated software based diagnosis. This concept has proven to be reliable for shallow tumors of $20 \mathrm{~mm}$ in diameter, but the analysis remains inconclusive for smaller inclusions.

This research presents a concept to detect these smaller inclusions, using principles from modal analysis theory, where the frequency response of a system with multiple degrees of freedom (MDOF) is considered to be the summation of the frequency responses corresponding to each of its modes [17, 18. Hypothesis testing is used to detect significant changes in the modal parameters which could indicate the presence of a tumor. 


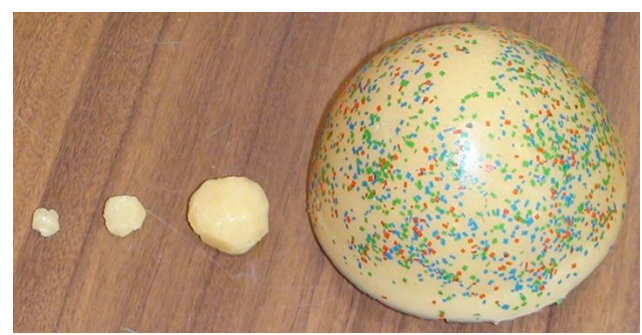

(a)

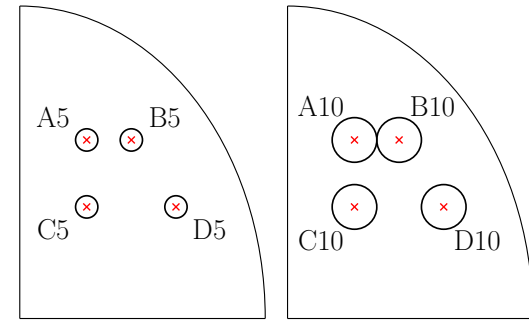

(b)

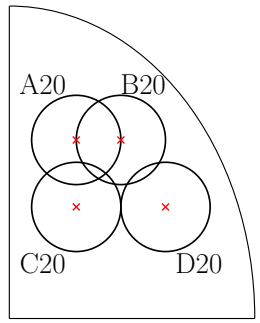

20

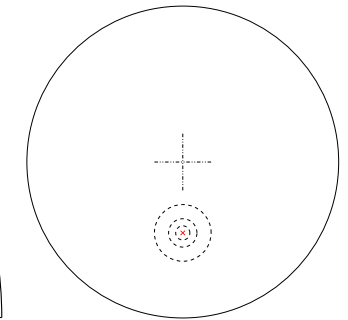

(c)

Figure 2: (a) Silicone breast phantom with examples of the stiffer inclusions placed inside, (b) the four locations (centrepoints in red) for the four tumor sizes (black circles), (c) tumor location B at the 6 -o'clock position in the breast when viewed from the front.

\section{Methodology}

\subsection{Experimental setup and data acquisition}

This DIET tumor detection concept study is performed on a set of thirteen silicone gel phantom breasts (A-341 Silicone, Factor2, Lakeside, USA) with comparable elastic properties to human tissue [10]. One of these phantoms is 'healthy', without any inclusions. The other twelve have inclusions of 5, 10 and 20 $\mathrm{mm}$ in diameter located at four different locations (depths) within the breast, all at a six-o'clock position when viewed from the front, as can be seen in Figure 2 .

Imaging of a breast is performed with the DIET clinical prototype. To enable robust tracking of the surface motion, randomly placed colored fiducial markers are applied [14] as can also been seen in Figure 2. Prior to imaging, the cameras are calibrated and provide 3D motion data of every tracked marker [14]. The tracked motion paths resemble an elliptical path in 3D-space. A sine curve, as in equation (1), is fit to these paths to determine the amplitude $A$ and phase $\phi$ in 3D for every marker's displacement $\bar{x}(t)[10$,

$$
\bar{x}(t)=\bar{A} \sin (\omega t+\bar{\phi})
$$

where $\bar{A}=\left[A_{x}, A_{y}, A_{z}\right]^{T}$ and $\bar{\phi}=\left[\phi_{x}, \phi_{y}, \phi_{z}\right]^{T}$. Similar to the analysis in [15], this analysis uses the displacement data in the direction of actuation, as characterized by $A_{z}$ and $\phi_{z}$.

A spherical coordinate system is used to describe the location of the markers as shown in Figure 3 . Angle $\alpha$ is the elevation angle with $0^{\circ}$ being at the chest wall and $90^{\circ}$ at the nipple, and $\theta$ is the rotational angle, starting with $0^{\circ}$ at the 12 -o'clock position when viewed from the front, rotating clockwise. To account for uneven marker coverage, the surface of the breast in discretized into intervals of $\alpha$ and $\theta$ and 


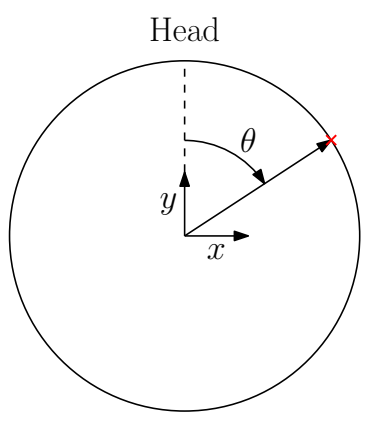

Feet

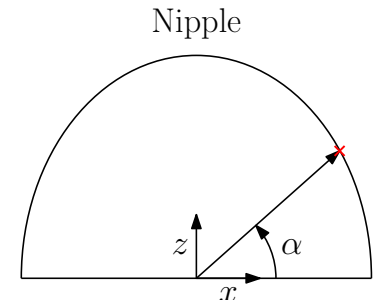

Chest wall

Figure 3: Definition of angles $\alpha$ and $\theta$. Angle $\alpha$ is the elevation angle with $0^{\circ}$ being at the chest wall and $90^{\circ}$ at the nipple, and $\theta$ is the rotational angle, starting with $0^{\circ}$ at the $12-0^{\prime}$ clock position when viewed from the front, rotating clockwise.
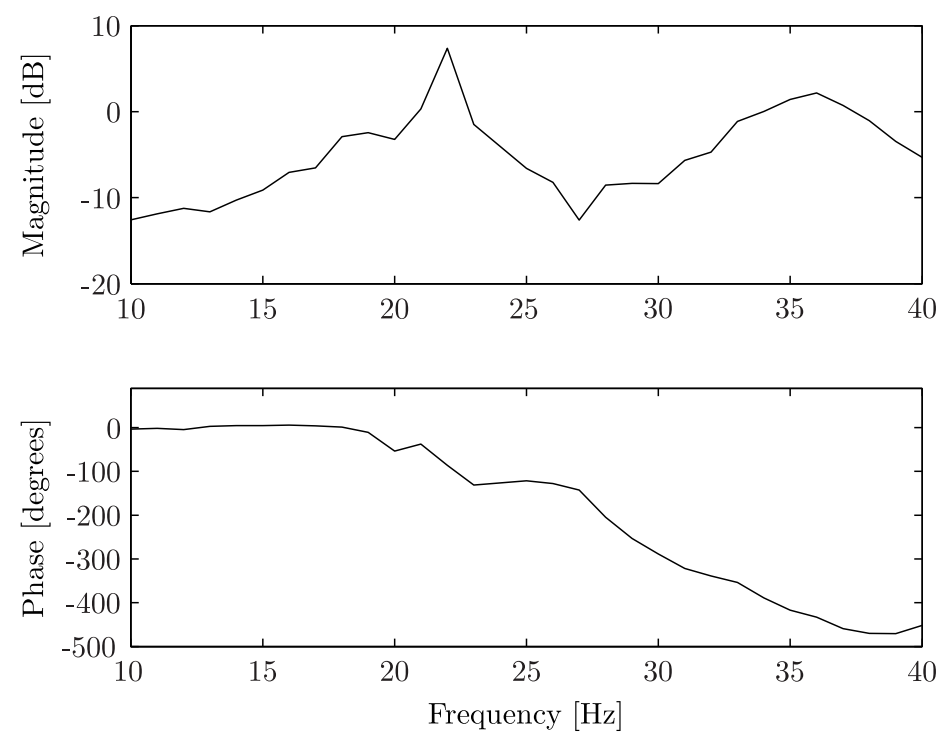

Figure 4: A typical Bode diagram for one of the segments of an imaged breast showing the first two natural frequencies.

the mean values of all tracked markers are used for each segment.

The silicone phantoms are imaged at frequencies between 10 and $40 \mathrm{~Hz}$ with a resolution of $1 \mathrm{~Hz}$ and Bode diagrams of the frequency response with respect to the actuator are made, covering the first and the second natural frequencies. A typical Bode diagram for one of the segments can be seen in Figure 4.

\subsection{Modal parameter estimation}

For a linear system with $n$ DOF, the equations of motion are known to be

$$
M \ddot{q}(t)+B \dot{q}(t)+K q(t)=f(t)
$$

where $q(t)$ is the displacement vector of length $n, f(t)$ the load vector of length $n$ and $M, B$ and $K$ are $n \times n$ mass, damping and stiffness matrices. Under the assumption of proportional damping, i.e. the condition 
$K M^{-1} B=B M^{-1} K$ holds, equation (2) can be decoupled using the matrix of eigenmodes $U_{O}$. By substituting the coordinate transformation $q(t)=U_{O} p(t)$ in equation 2 followed by a premultiplication with $U_{O}{ }^{T}$, the decoupled equations of motion result in:

$$
U_{O}^{T} M U_{O} \ddot{p}(t)+U_{O}^{T} B U_{O} \dot{p}(t)+U_{O}^{T} K U_{O} p(t)=U_{O}^{T} f(t)
$$

When the matrix of eigenmodes $U_{O}$ is mass normalized, i.e. $U_{O}{ }^{T} M U_{O}=I$, equation (3) transforms into

$$
I \ddot{p}(t)+2 \Xi \Omega_{0} \dot{p}(t)+\Omega_{0}^{2} p(t)=U_{O}^{T} f(t)
$$

where $\Xi$ is a diagonal matrix with dimensionless modal damping factors $\xi_{k}$, and $\Omega_{0}$ is a diagonal matrix with undamped natural frequencies $\omega_{O k}$.

Under the assumption of harmonic excitation and response and using the back-substitution of the coordinate transformation $q(t)=U_{O} p(t)$, the frequency response matrix obtained from equation (4) becomes:

$$
\begin{aligned}
\hat{q}(\omega) & =U_{O}\left(-\omega^{2} I+2 j \omega \Xi \Omega_{0}+\Omega_{0}^{2}\right)^{-1} U_{O}^{T} \hat{f}(\omega) \\
& =\sum_{k=1}^{n} \frac{u_{O k} u_{O k}{ }^{T}}{-\omega^{2}+2 \xi_{k} \omega_{O k} \omega j+\omega_{O k}^{2}} \hat{f}(\omega)
\end{aligned}
$$

Now, in the case of a single-input-single-output (SISO) MDOF system, equation (5) reduces to a summation of single mass-spring-damper systems multiplied with a constant which is determined by the modes of the system. As this tumor detection concept only takes the first two natural frequencies into account, equation (5) can be written as

$$
\hat{q}(\omega)=\frac{A_{1}}{-\omega^{2}+2 \xi_{1} \omega_{O 1} \omega j+\omega_{O 1}^{2}}+\frac{A_{2}}{-\omega^{2}+2 \xi_{2} \omega_{O 2} \omega j+\omega_{O 2}^{2}}
$$

where $A 1$ and $A 2$ are constant multiplication factors determined by the eigenmodes of the system.

In this way, the problem of fitting the data in the Bode diagram in Figure 4 can be reduced and the data can be split in two to fit each natural frequency independent from the other.

Quality of the model fits discussed, is objectively assessed by taking the root mean square (RMS) deviation $e$ of a fits magnitude, say $\hat{A}$, compared to the magnitude of the measurement data, say $A$, using $n$ datapoints, defined by:

$$
e=\sqrt{\frac{1}{n} \sum_{i=1}^{n}\left(\hat{A}_{i}-A_{i}\right)^{2}}
$$

Fitting the two peaks at once proved to be hard using nonlinear least squares methods. Although the model structure is capable of capturing the response as given in Figure 4 , verified by manual parameter estimation, these nonlinear least squares methods ended up in local minima or identified 'false' peaks due to noise in the data. A typical example of these bad fits is given in Figure 5 , with an $e$-value of 0.66. The two estimated natural frequencies for this segment are 19.2 and $22.4 \mathrm{~Hz}$, where the first one is not visible due to a very small gain. The $19.2 \mathrm{~Hz}$ frequency is obviously wrong and is probably triggered by the noise in the data, finding a local minimum instead of a global minimum. To overcome this the 

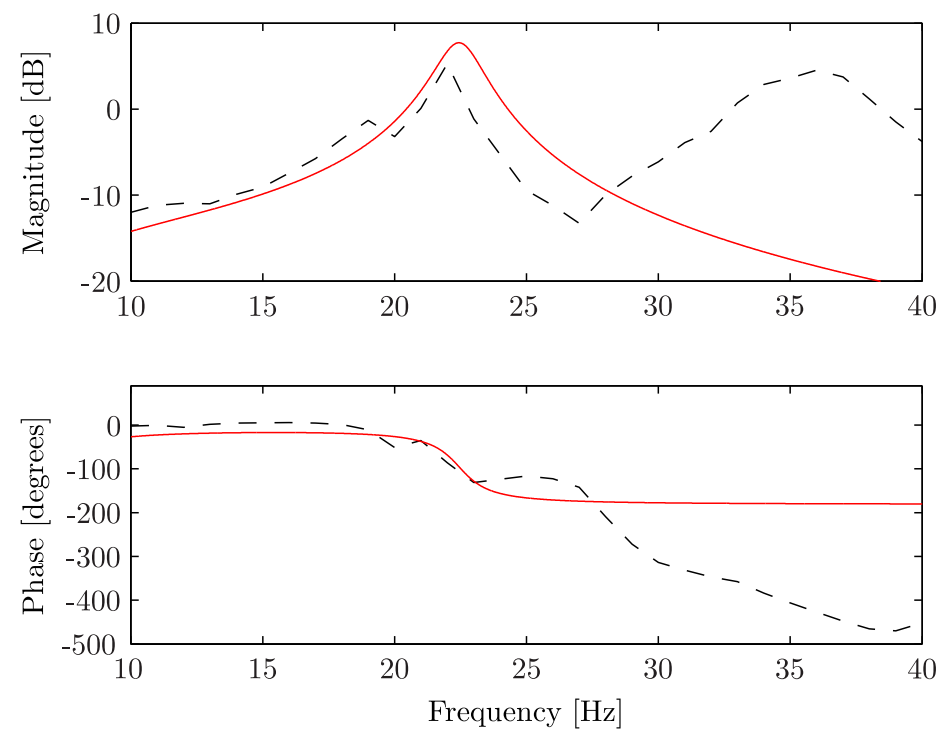

Figure 5: Typical example of a problem with using a nonlinear least squares method. The two estimated natural frequencies in this case are 19.2 and $22.4 \mathrm{~Hz}$, where the first one is not visible due to a very small gain. This effect is probably triggered by the noise in the data.

problem, the data is split into two parts and is fitted independently.

First, the end of the first peak is determined, under the assumption that the end of this peak is characterized by a local minimum in the magnitude and a phase of $180^{\circ}$. Robustness margins are incorporated to ensure reliable results with noisy data.

The fitting procedure as described in [15] is used to determine the first natural frequency $\omega_{O 1}$ and the corresponding modal damping factor $\xi_{1}$, using data up to the end of the first peak. This method proved to be very robust and reliable.

The second natural frequency is fitted using the data for frequencies larger than the first natural frequency $\omega_{O 1}$. Here, the entire model structure as in equation (6) is used to assure a good match in phase. This produces reliable results for the second natural frequency and corresponding damping factor. The first natural frequency is estimated wrongly, because only the downward slope of the first peak is 'visible' to the fitting procedure. By combining the results from the two fitting procedures, good and reliable fits are obtained with $e$-values of approximately 0.2 , allowing further analysis on the modal parameters, especially the second natural frequency $\omega_{\mathrm{O} 2}$, which proves to be a reliable metric to detect tumor location.

\section{$2.3 \quad$ Statistical analysis}

The analysis is performed with 18 segments in $\theta$-direction and 7 segments in $\alpha$-direction, moving from $15^{\circ}$ to $45^{\circ}$ to exclude misfits around the actuator and around the chest wall. The actuator and the chest wall disturb the surface motion of the breast yielding a different type of response. Increasing the number of segments might give a higher resolution, but also increases the chance of gaps in the data due to gaps in the fiducial marker coverage. Figure 6 shows an example of the used segments overlayed upon a triangulation of the tracked fiducial markers.

To analyze and detect any significant changes of this metric within the breast, statistical hypothesis 


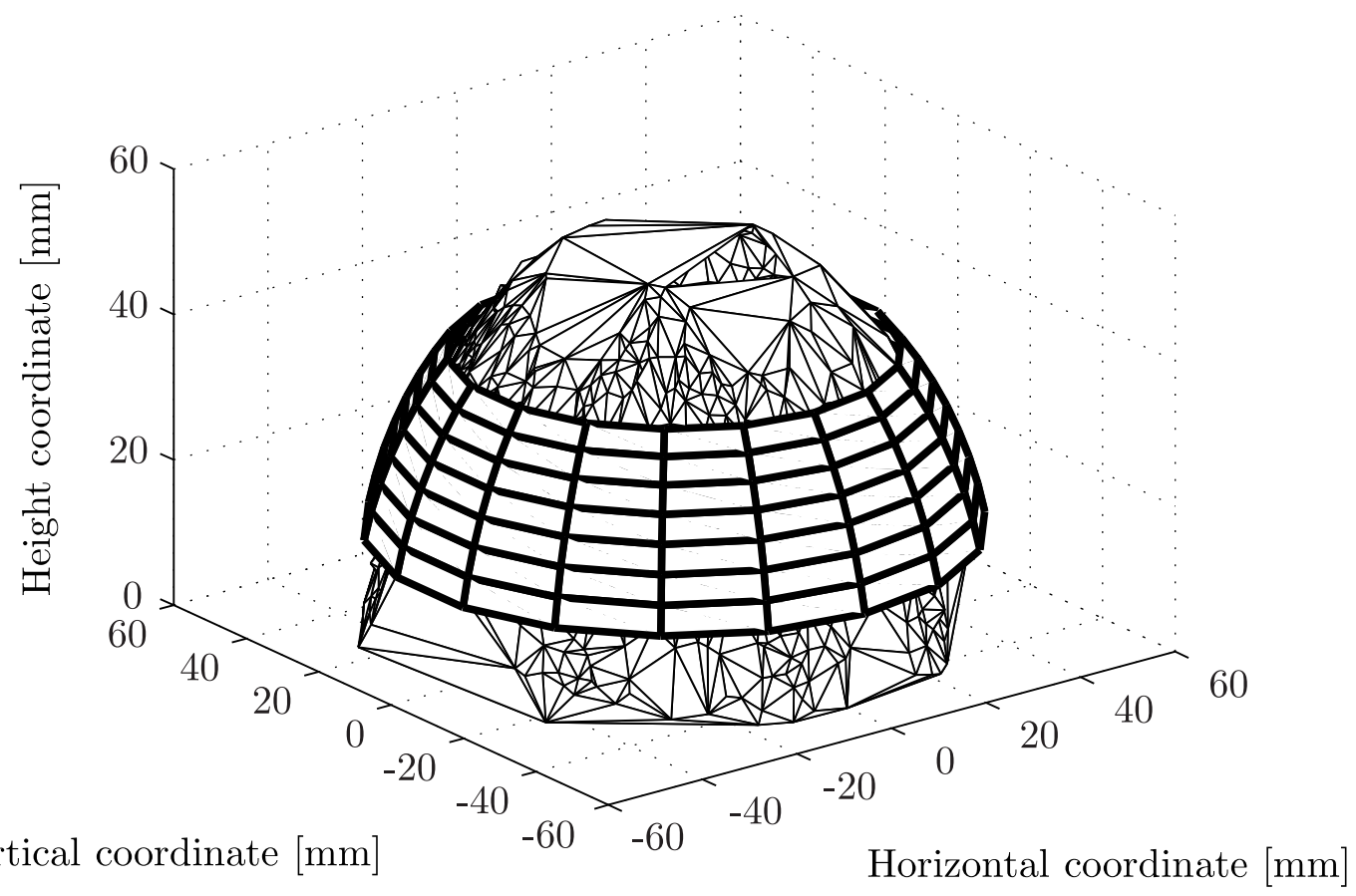

Figure 6: An example of the used segments overlayed upon a triangulation of the tracked fiducial markers.

testing is applied. The segments of the breast are divided into two groups. The first group includes all segments within a $60^{\circ}$ range in $\theta$-direction. The second group consists of all the other segments. This is repeated for all $18 \theta$-segments.

A two-sample $t$-test is used to compare both groups, where the null hypothesis is that both groups are from the same normal distributions with equal means and unequal variances. The alternative hypothesis is that the first group is from a different normal distribution with a lower mean value. An $\alpha$-level of 0.01 is chosen, indicating a $99 \%$ confidence rate.

To furthermore assess statistical significance, the probability of obtaining the data in the analyzed segment or lower, assuming that the null hypothesis indeed is true, is calculated and is called the $p$-value of the test. A segment with a low $p$-value, specifically a $p$-value below the $\alpha$-level, indicates a signifact lower second natural frequency in that section.

\section{Results}

The method discussed above is applied to all thirteen silicone phantoms. Figure 7 shows a typical example of the fitting procedure. Separate fits for the first and second natural frequency are shown and their sum produces a close match to the original measurement data.

In Figure 8 as segmented breast when viewed from the front, is overlayed with a colormap of the first natural frequency $\omega_{O 1}$ for phantom B20 as indicated in Figure 2b. The gaps in the colormap represent bad model fits due to noise and gaps in the data. At the tumor location (6-o'clock position) an area with a significant higher first natural frequency is clearly visible, with an increase of about $3 \mathrm{~Hz}$. This is the effect as reported in [15. This distinctive behavior occurs in all phantoms with tumors that are 20 


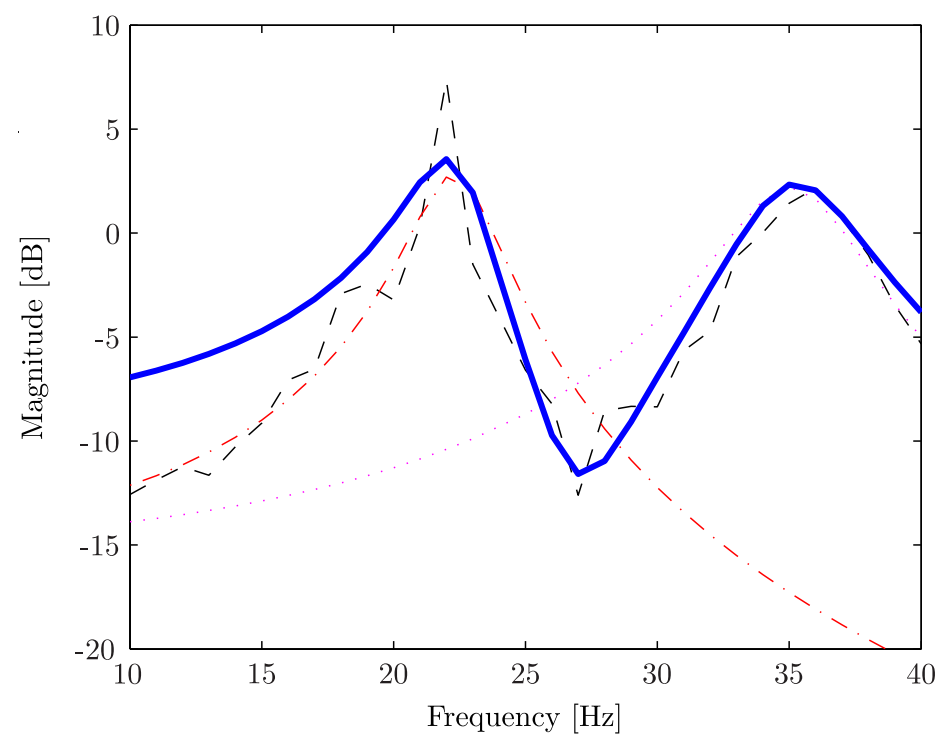

Figure 7: A typical example of the fitting results. Measurement data in dashed black, fit of the first natural frequency in dash-dotted red, fit of the second natural frequency in dotted magenta and the combined fit in solid blue.

$\mathrm{mm}$ in diameter.

In the healthy phantom, shown in Figure 9, the clear distinctive behavior is not present, but only a slight gradual variation of about $1 \mathrm{~Hz}$ is visible. In the phantoms with smaller tumors, for instance phantom B10 as shown in Figure 10, no distinctive behavior can be found and the results are similar to the healthy case, with a $1 \mathrm{~Hz}$ gradual variation. The first natural frequency is analyzed for all phantoms and the results can be found in Figures 16 28 in Appendix A,

Figure 11 shows the second natural frequency $\omega_{O 2}$. The analyzed phantom in this case is B10 as indicated in Figure 2b. At the 6-o'clock position, an area with a smaller second natural frequency is clearly visible. Similar effects are visible in all phantoms with 10 and $20 \mathrm{~mm}$ tumors. These effects are not visible in the phantoms without inclusion and in the phantoms with $5 \mathrm{~mm}$ tumors. Figures 29 41 in Appendix B show the second natural frequency $\omega_{O 2}$ for all analyzed phantoms.

To analyze the differences in the second natural frequency objectively, Figure 12 shows the probability density functions for the normal distributions in two different segments of the breast, again for a B10 phantom. The black lines represent the distributions for the range $300^{\circ}<\theta<360^{\circ}$ (top of the breast) and the red lines represent the distribution for the range $160^{\circ}<\theta<220^{\circ}$ around the location of the tumor. The solid lines represent the data in the given ranges, the dashed lines correspond to all the data outside this range. Clearly, the data with $160^{\circ}<\theta<220^{\circ}$ (solid red) has a different mean than the data outside this region (dashed red). In the second case, the two distributions corresponding to the data within the $300^{\circ}<\theta<360^{\circ}$ range (solid black) and the data outside this range (dashed black), show a great amount of overlap.

To further verify these results, Figure 13 shows the result of the statistical $t$-test. The red area (value 1 ) is the area where the null hypothesis is rejected, indicating a lower second natural frequency with a $99 \%$ confidence level. The average $p$-value in the red area equals $6.486 \cdot 10^{-7}$, while the average $p$-value outside this area yields 0.8521 . 


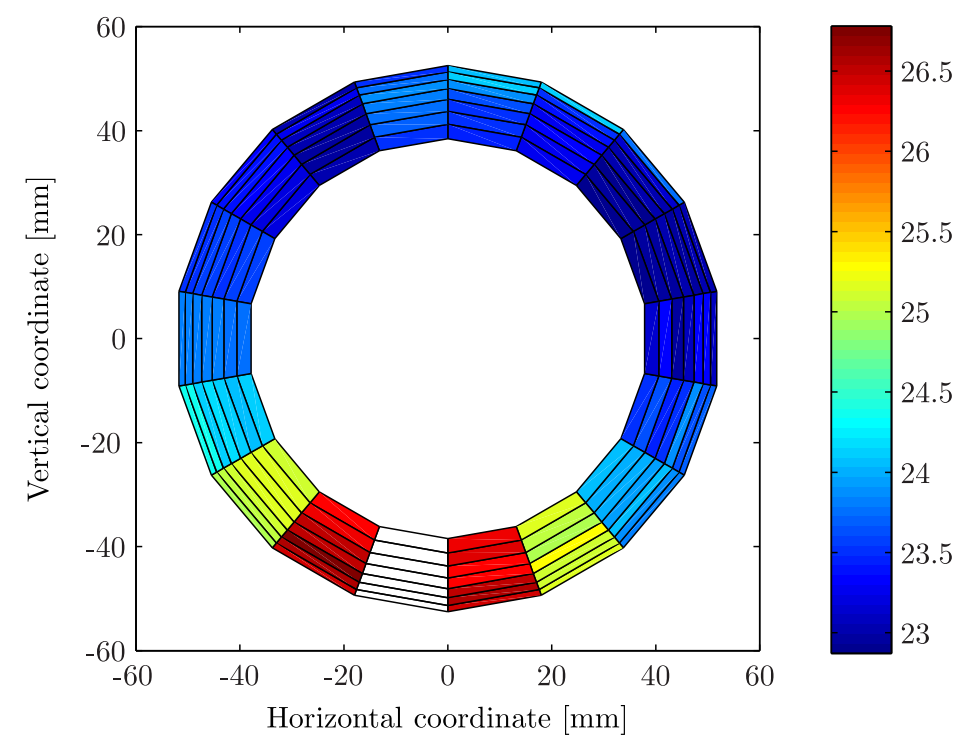

Figure 8: Segmented breast when viewed from the front with a colormap of the first natural frequency $\omega_{O 1}$ in $[\mathrm{Hz}]$ for a B20 silicone phantom as indicated in Figure 2b. Blank sections indicate bad model fits due to noise and gaps in the data.

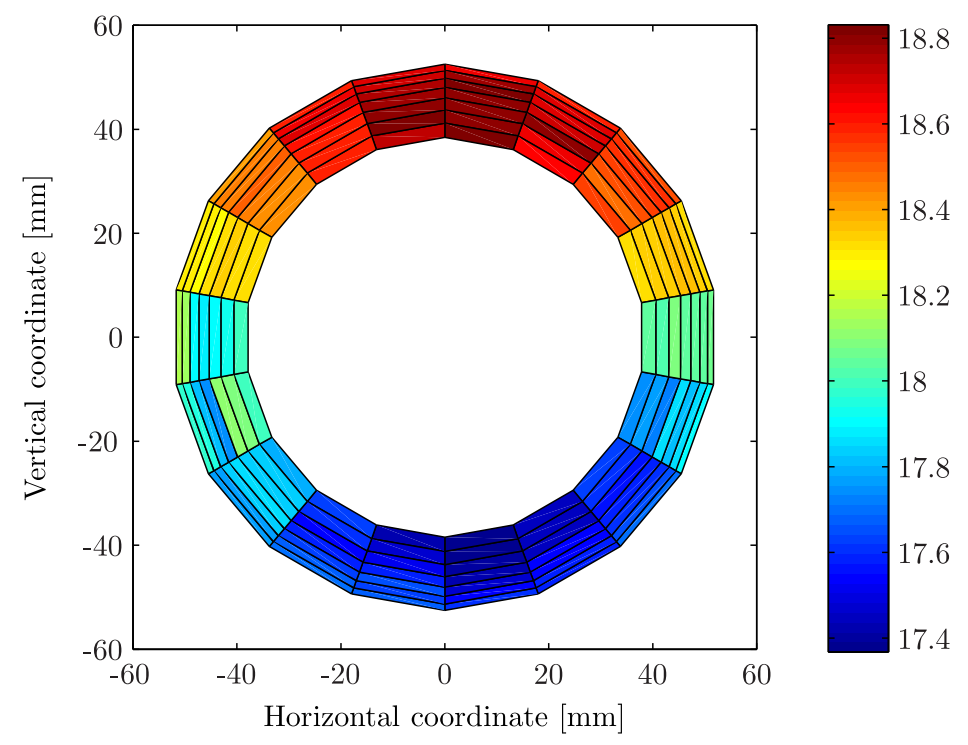

Figure 9: Segmented breast when viewed from the front with a colormap of the first natural frequency $\omega_{O 1}$ in $[\mathrm{Hz}]$ for a healthy silicone phantom. 


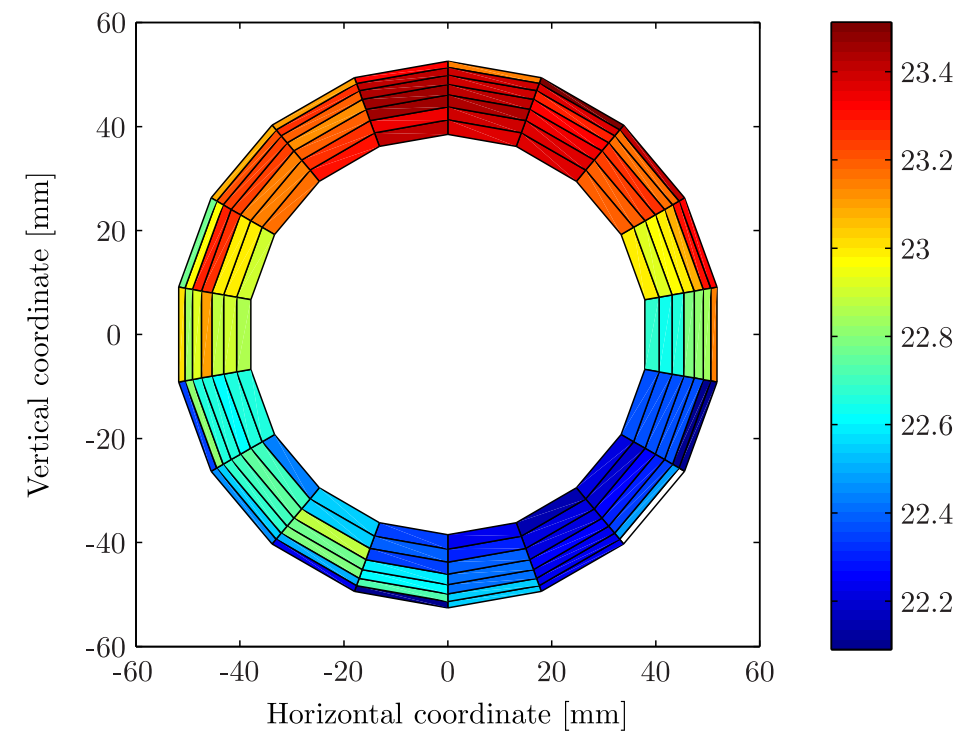

Figure 10: Segmented breast when viewed from the front with a colormap of the first natural frequency $\omega_{O 1}$ in $[\mathrm{Hz}]$ for a $\mathrm{B} 10$ silicone phantom as indicated in Figure $2 \mathrm{~b}$.

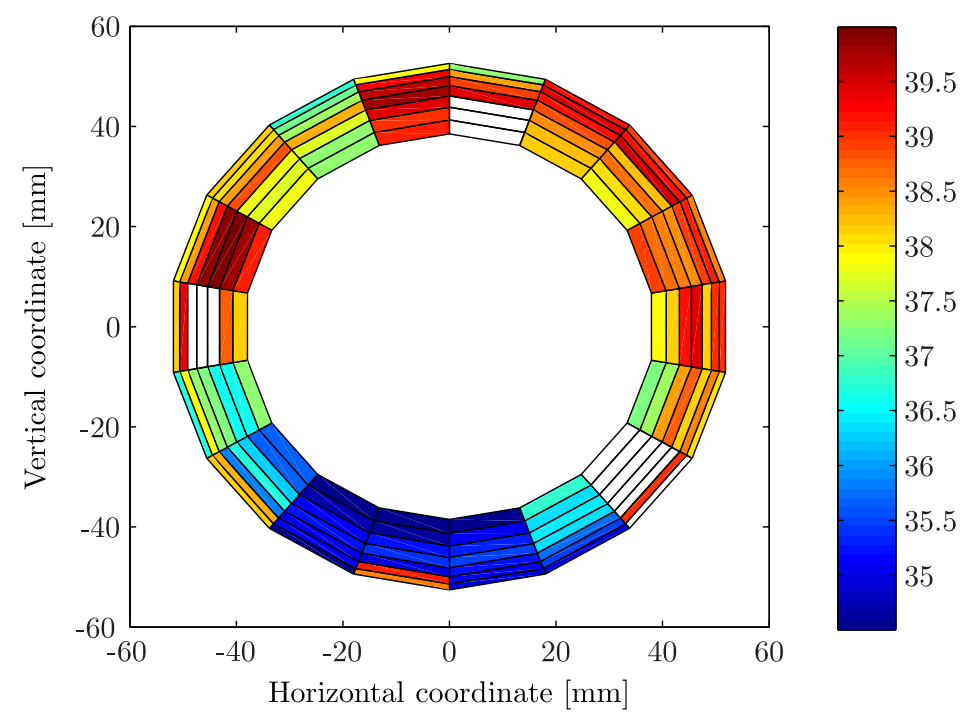

Figure 11: Segmented breast when viewed from the front with a colormap of the second natural frequency $\omega_{O 2}$ in $[\mathrm{Hz}]$ for a B10 silicone phantom as indicated in Figure 2b. Blank sections indicate bad model fits due to noise and gaps in the data. 


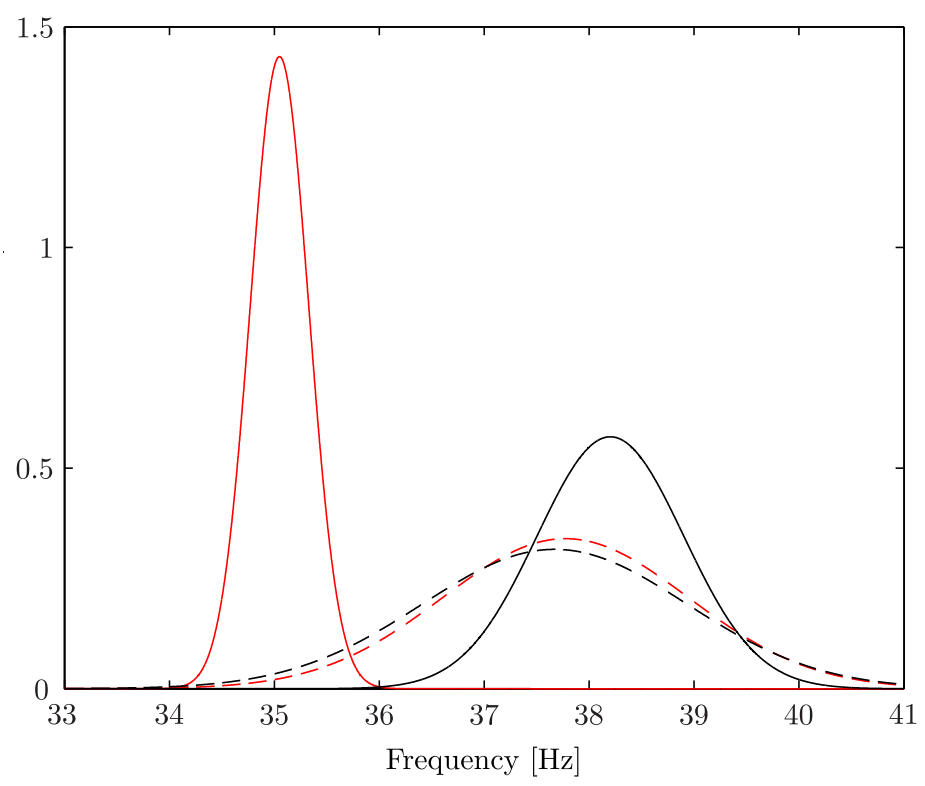

Figure 12: Probability density functions for the normal distributions in two different segments of the breast of a B10 phantom. The solid red line represents the data in the segment $160^{\circ}<\theta<220^{\circ}$ and the dashed red line corresponds to data outside this segment. Similar, the solid black line show data in the segment $300^{\circ}<\theta<360^{\circ}$ where the dashed black line considers the data outside this range.

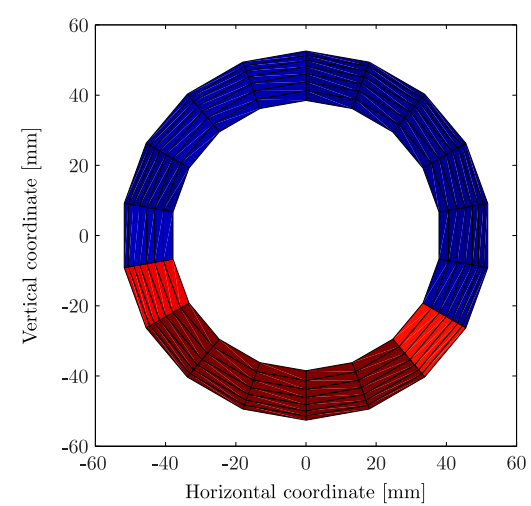

(a)
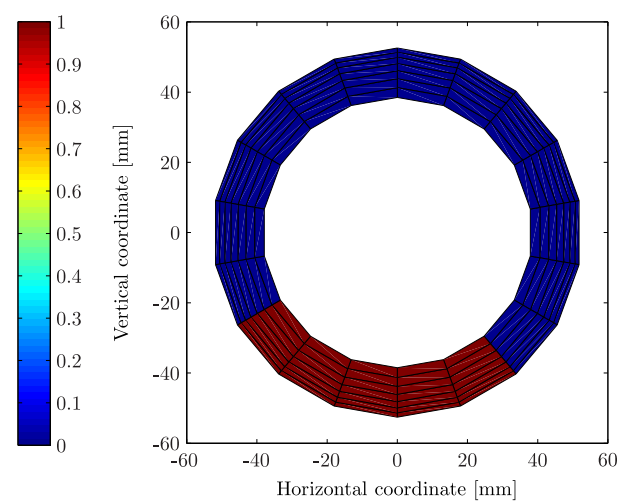

(b)

Figure 13: Result of the $t$-test for the B10 phantom, overlayed on the segmented breast. The metric $1-p$ is shown on the left (a), such that dark red resembles a low $p$-value and possibly a tumor. The test outcome is given on the right (b), where the red area indicates rejection of the stated null hypothesis, indicating a lower second natural frequency and thus a stiffer inclusion, and the blue area represents acceptance of the null hypothesis. 
Table 1: Results of the $t$-test in terms of $p$-values for all thirtheen segment. A comparison is made between the area where the null hypothesis is rejected (e.g. red area in Figure 13b), possibly an indication for an inclusion, and the area where the null hypothesis is accepted (e.g. blue area in Figure 13b.

\begin{tabular}{|l|l|l|}
\hline Phantom: & $\begin{array}{l}\text { Average } p \text {-value in } \\
\text { rejection area }\end{array}$ & $\begin{array}{l}\text { Average } p \text {-value in } \\
\text { acceptance area }\end{array}$ \\
\hline \hline Healthy & - & 0.50653 \\
\hline A5 & $4.5213 \cdot 10^{-4}$ & 0.67531 \\
\hline B5 & $1.6714 \cdot 10^{-3}$ & 0.63221 \\
\hline C5 & $1.5228 \cdot 10^{-3}$ & 0.57836 \\
\hline D5 & $7.4005 \cdot 10^{-3}$ & 0.50929 \\
\hline A10 & $1.5059 \cdot 10^{-3}$ & 0.89132 \\
\hline B10 & $6.7370 \cdot 10^{-7}$ & 0.80685 \\
\hline C10 & $2.5711 \cdot 10^{-3}$ & 0.68937 \\
\hline D10 & $3.7114 \cdot 10^{-3}$ & 0.57493 \\
\hline A20 & $1.5344 \cdot 10^{-3}$ & 0.72851 \\
\hline B20 & $1.5399 \cdot 10^{-4}$ & 0.87132 \\
\hline C20 & $4.3793 \cdot 10^{-4}$ & 0.66437 \\
\hline D20 & $2.2566 \cdot 10^{-8}$ & 0.63102 \\
\hline
\end{tabular}

For all thirtheen phantoms, the $p$-values in the area where the null hypothesis is rejected are compared to the $p$-values in the area where the null hypothesis is accepted by taking the average $p$-value within the stated areas. The results can be found in Table 1 .

All discussed results show similar behavior in all phantoms with 10 and $20 \mathrm{~mm}$ tumors. Furthermore, the 'healthy' phantom ( $t$-test results shown in Figure 14) does not show this response. Results for the $5 \mathrm{~mm}$ tumors remain inconclusive under this method. Figure 15 shows the results for phantom D5, yielding a false positive. Figures 2941 in Appendix B show the results of the $t$-test for all analyzed phantoms.

\section{Discussion}

The proposed method using the model in equation (6) produced good model fits. The model is able to capture the frequency response data from the surface of the oscillating breast phantom. By splitting the problem in two parts, the method as discussed in [15] can be used, which proves to be very robust and reliable. The fitting of the second peak gives some problems for the segments close to the chest wall and close to the actuator, but produces good fits in the other segments.

As described in [15] the first natural frequency is a reliable metric to detect tumors of $20 \mathrm{~mm}$ in diameter, with significant behavior at the location of the tumor. However, for smaller inclusions, the results remain inconclusive.

The second natural frequency identified over the surface of the breast shows a distinctive decrease around the tumor location. For all phantoms with 10 and $20 \mathrm{~mm}$ inclusion, this distinctive behavior shows up and it remains unseen in the phantom without any inclusions. Detection of a $10 \mathrm{~mm}$ inclusion, which is clinically significant in a screening application [19], is possible with this method. Tumors of $5 \mathrm{~mm}$ in diameter also show no clear distinctive behavior. Imaging the breast up to higher frequencies and incorporating the third natural frequency might give conclusive results for even smaller inclusions, thus being a lead for future research. 


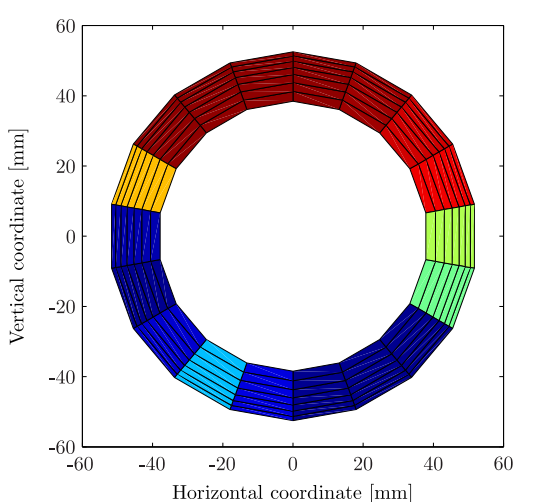

(a)
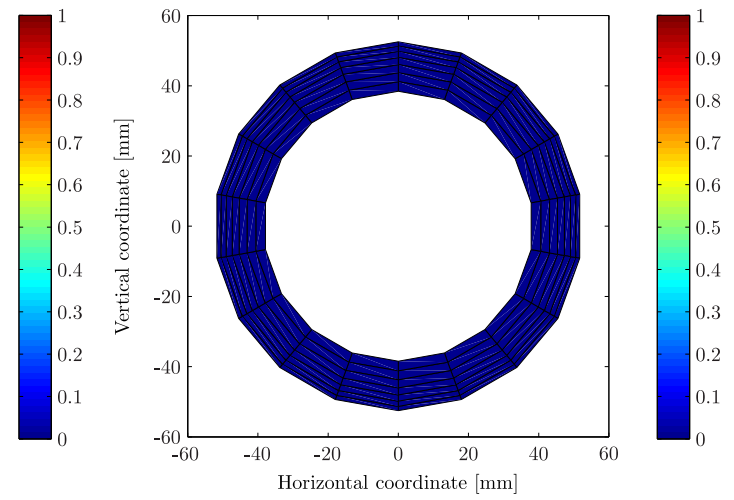

(b)

Figure 14: Result of the $t$-test for the healthy phantom, overlayed on the segmented breast. The metric $1-p$ is shown on the left (a), such that dark red resembles a low $p$-value and possibly a tumor. The test outcome is given on the right (b), where the blue area represents acceptance of the null hypothesis. In this case, no rejection is present, indicating no inclusions.

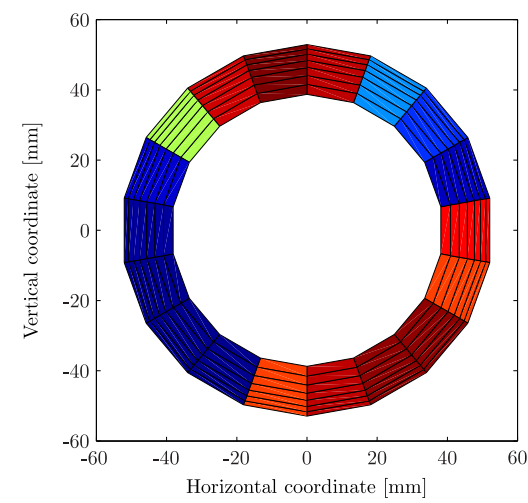

(a)
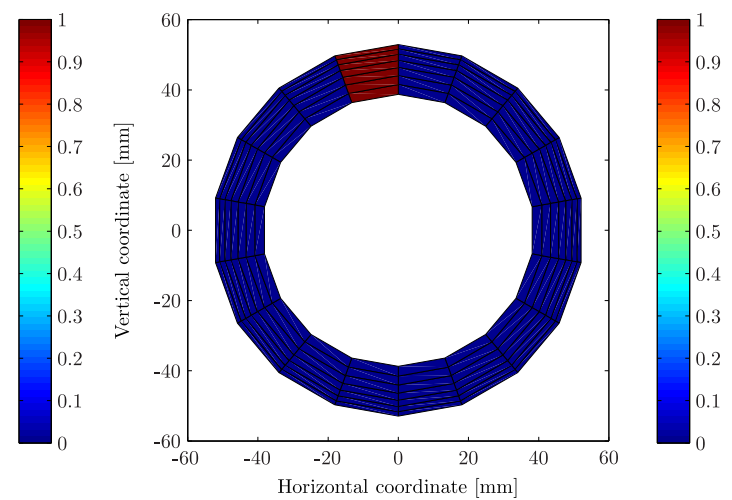

(b)

Figure 15: Result of the $t$-test for the B10 phantom, overlayed on the segmented breast. The metric $1-p$ is shown on the left (a), such that dark red resembles a low $p$-value and possibly a tumor. Notice that in this case, two areas with low $p$-values show up. The test outcome is given on the right (b), where the red area indicates rejection of the stated null hypothesis, and the blue area represents acceptance of the null hypothesis. Only one of the two areas with low $p$-values is low enough to reject the null hypothesis. 
The distinctive behavior is verified by statistical analysis, with a less than $1 \%$ change that this behavior is not caused by a stiffer inclusion, but by chance. The fact that the results are consistent for all 10 and $20 \mathrm{~mm}$ inclusions further indicates that the results are not coincidental.

For some of the phantoms, the identified inclusion area shows a deviation with the exact tumor location. This is a known effect and is caused by assymetrical imaging of the particular phantom. FEM simulations of exactly the same situation (mesh based on measured surface points of the phantom) show a similar deviation in the disturbance of the motion data. Deviations up to $45^{\circ}$ are considered to be acceptable.

Furthermore, in the outcome of the $t$-test a difference in size of the region where the null hypothesis is rejected, is notable. Presumably, this effect can be a further lead for the location of the tumor inside the breast in terms of distance from the surface. Phantoms D10 and D20, closest to the analyzed part of the surface, show smaller regions of rejectance, which might be caused by a very localized effect due to an inclusion which is very close to the surface and thus not affecting large areas of this surface. Tumors deeper inside the breast show larger regions of rejectance, possibly due to the fact that deeper tumors affect larger areas on the surface of the breast. Further research is necessary to assess the validity of this phenomenon, both in vitro and in vivo.

This analysis only uses the surface motion in the direction of actuation. However, the surface of the breast moves in 3D-space. Therefore, multidimensional models might be useful to further enhance the tumor detection algorithm for the DIET system.

Overall, the concept needs further in vivo research to validate the results in real, human breasts. It is expected that there is a limit on the tumor size that can be detected with the DIET system, but manual analysis on the measurement data shows small disturbances in the measurement data for the $5 \mathrm{~mm}$ tumors. Further research should focus on finding automated analysis algorithms to detect these smaller inclusions.

\section{Conclusion}

The presented approach is capable of detecting any tumors of $10 \mathrm{~mm}$ in diameter in larger in silicone phantoms in an objective and repeatable way and is computationally inexpensive, especially when compared to inverse finite element analysis. When in vivo validation yields similar results, the presented method can be used to detect the existence and approximate location of a stiffer inclusion. This method provides software based diagnosis without any human intervention, yielding low operator skill requirements. Combined with the DIET system, this approach could provide a reliable screening modality, complementary to mammography and other currently used screening techniques.

\section{References}

[1] S. S. Coughlin and D. U. Ekwueme. Breast cancer as a global health concern. Cancer epidemiology, 33(5):315-8, 2009.

[2] Daniel B. Kopans. Breast imaging. Lippincott Williams \& Wilkins, Philadelphia, 2nd edition, 1998.

[3] Cancer patient survival covering the period 1994 to 2003. Technical report, New Zealand Ministry of Health, 2006.

[4] M. Kriege, C. T. Brekelmans, C. Boetes, P. E. Besnard, H. M. Zonderland, I. M. Obdeijn, R. A. Manoliu, T. Kok, H. Peterse, M. M. Tilanus-Linthorst, S. H. Muller, S. Meijer, J. C. Oosterwijk, L. V. Beex, R. A. Tollenaar, H. J. de Koning, E. J. Rutgers, and J. G. Klijn. Efficacy of mri and mammography for breast-cancer screening in women with a familial or genetic predisposition. The New England journal of medicine, 351(5):427-37, 2004. 
[5] R. Fazel, H. M. Krumholz, Y. Wang, J. S. Ross, J. Chen, H. H. Ting, N. D. Shah, K. Nasir, A. J. Einstein, and B. K. Nallamothu. Exposure to low-dose ionizing radiation from medical imaging procedures. The New England journal of medicine, 361(9):849-57, 2009.

[6] A. Asghari and M. K. Nicholas. Pain during mammography: the role of coping strategies. Pain, 108(1-2):170-9, 2004.

[7] P. C. Gotzsche, O. J. Hartling, M. Nielsen, J. Brodersen, and K. J. Jorgensen. Breast screening: the facts-or maybe not. BMJ (Clinical research ed, 338:b86, 2009.

[8] A. Sarvazyan, V. Egorov, J. S. Son, and C. S. Kaufman. Cost-effective screening for breast cancer worldwide: Current state and future directions. Breast cancer, 1:91-99, 2008.

[9] L. Yang, D. M. Parkin, J. Ferlay, L. Li, and Y. Chen. Estimates of cancer incidence in china for 2000 and projections for 2005. Cancer epidemiology, biomarkers \& prevention, 14(1):243-50, 2005.

[10] T. Lotz, P. D. Simpson, D. Stocker, C. E. Hann, and J. G. Chase. In vitro evaluation of surface based non-invasive breast cancer screening with digital image based elasto tomography (diet). In IEEE EMBS, volume 1, pages 3077-80, Buenos Aires, 2010.

[11] A. Samani, J. Zubovits, and D. Plewes. Elastic moduli of normal and pathological human breast tissues: an inversiontechnique-based investigation of 169 samples. Physics in medicine and biology, 52(6):1565-76, 2007.

[12] A. Peters, J. G. Chase, and E. E. Van Houten. Estimating elasticity in heterogeneous phantoms using digital image elasto-tomography. Medical and biological engineering and computing, 47(1):67-76, 2009.

[13] R.G. Brown, C.E. Hann, J.G. Chase, and X.Q. Chen. Vision-based 3d surface motion capture for the diet breast cancer screening system. In 15th International Conference on Mechatronics and Machine Vision in Practice (M2VIP), pages 674-679, Auckland, New Zealand, 2008 of Conference.

[14] R.G. Brown, C.E. Hann, J.G. Chase, and L.E. Ray. Discrete colour-based euclidean-invariant signatures for feature tracking in a diet breast cancer screening system. In SPIE Medical Imaging, volume 6511, San Diego, USA, 2007 of Conference. SPIE.

[15] T. Lotz, N. Muller, C. E. Hann, and J. G. Chase. Minimal elastographic modeling of breast cancer for model based tumor detection in a digital image elasto tomography (diet) sytem. 2011.

[16] A. Peters, J. G. Chase, and E. E. Van Houten. Digital image elasto-tomography: combinatorial and hybrid optimization algorithms for shape-based elastic property reconstruction. IEEE transactions on bio-medical engineering, 55(11):2575-83, 2008.

[17] Jimin He and Zhi-Fang Fu. Modal analysis. Butterworth Heinemann, 2001.

[18] Tony L. Schmitz. Machining dynamics: frequency response to improved productivity. Springer US, 2009.

[19] J. S. Michaelson, M. Silverstein, J. Wyatt, G. Weber, R. Moore, E. Halpern, D. B. Kopans, and K. Hughes. Predicting the survival of patients with breast carcinoma using tumor size. Cancer, 95(4):713-23, 2002. 


\section{A First natural frequecny for all thirtheen phantoms}

Figures 1628 show the first natural frequency $\omega_{O 1}$ for the indicated phantom. The gaps in the colormap represent bad model fits due to noise and gaps in the data.

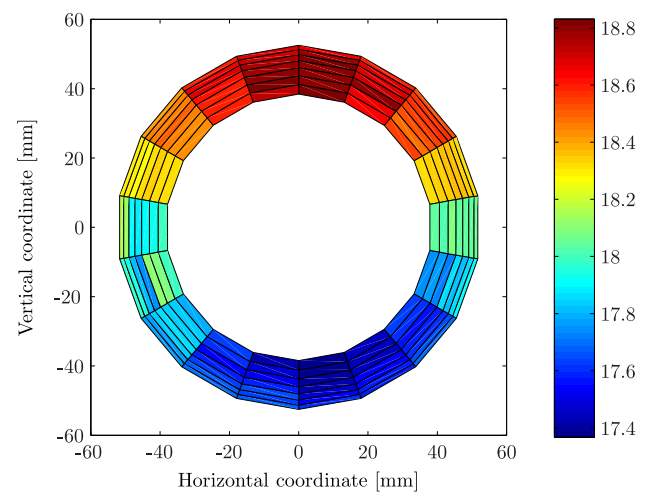

Figure 16: First natural frequency $\omega_{O 1}$ in $[\mathrm{Hz}]$ for a healthy phantom.

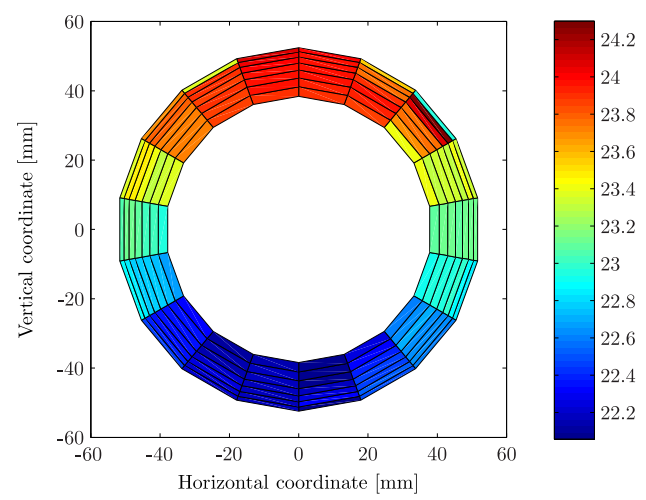

Figure 17: First natural frequency $\omega_{O 1}$ in $[\mathrm{Hz}]$ for a $\mathbf{A 5}$ phantom.

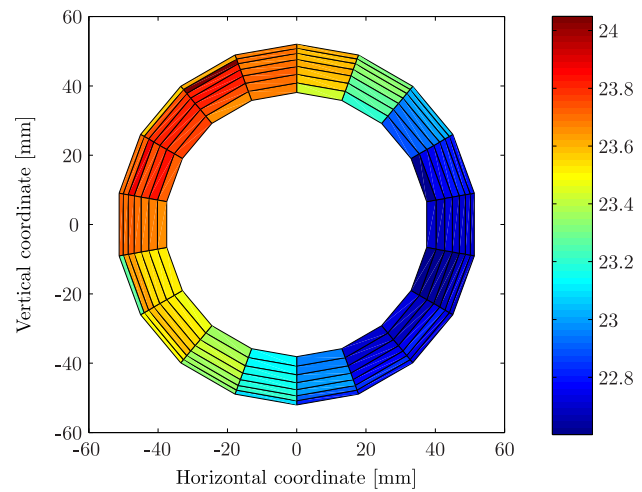

Figure 18: First natural frequency $\omega_{O 1}$ in $[\mathrm{Hz}]$ for a $\mathbf{B 5}$ phantom. 


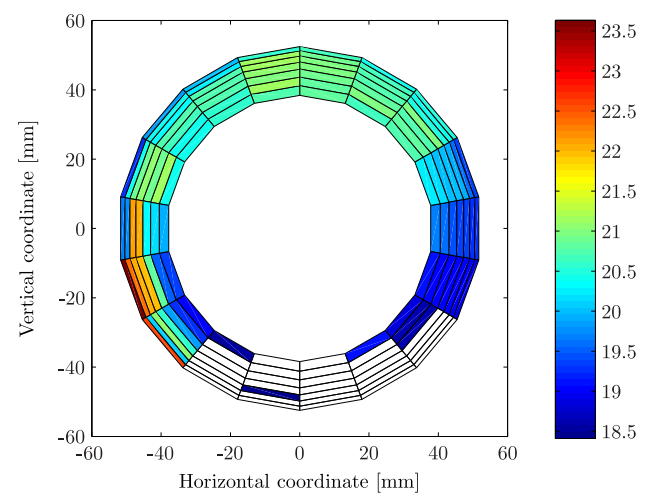

Figure 19: First natural frequency $\omega_{O 1}$ in $[\mathrm{Hz}]$ for a $\mathbf{C 5}$ phantom.

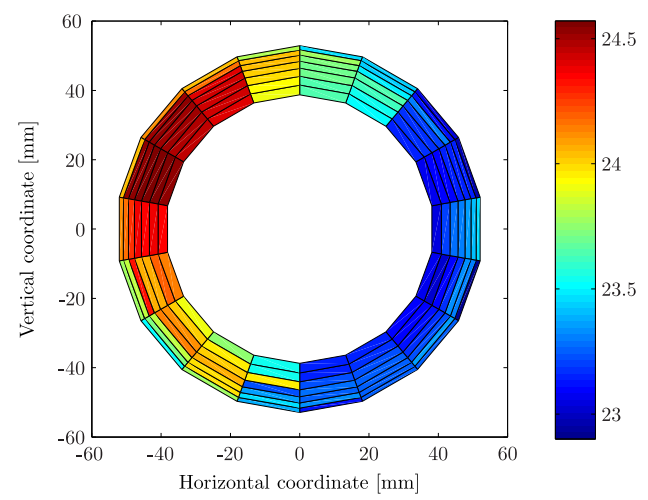

Figure 20: First natural frequency $\omega_{O 1}$ in $[\mathrm{Hz}]$ for a D5 phantom.

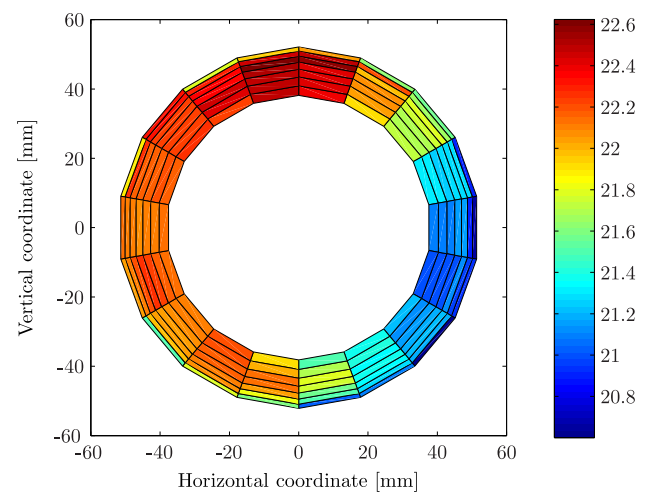

Figure 21: First natural frequency $\omega_{O 1}$ in $[\mathrm{Hz}]$ for a $\mathbf{A 1 0}$ phantom. 


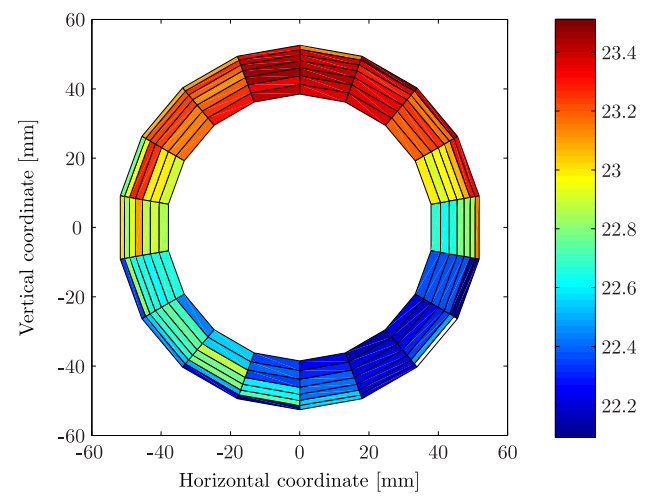

Figure 22: First natural frequency $\omega_{O 1}$ in $[\mathrm{Hz}]$ for a $\mathbf{B} 10$ phantom.

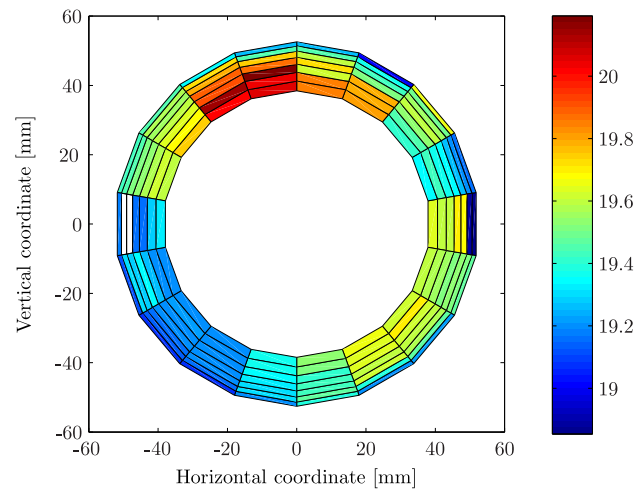

Figure 23: First natural frequency $\omega_{O 1}$ in $[\mathrm{Hz}]$ for a $\mathbf{C 1 0}$ phantom.

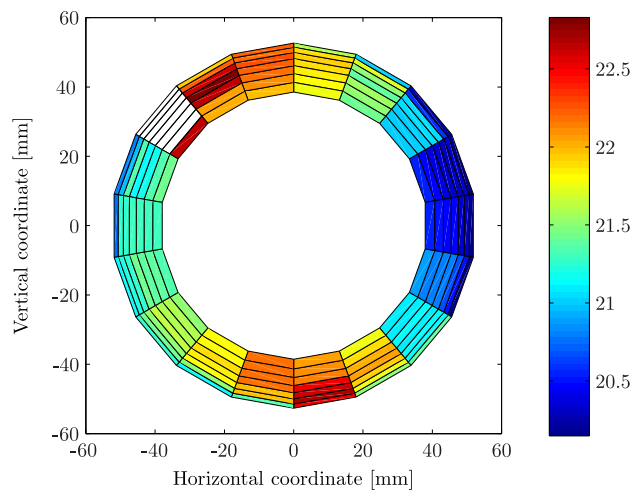

Figure 24: First natural frequency $\omega_{O 1}$ in $[\mathrm{Hz}]$ for a D10 phantom. 


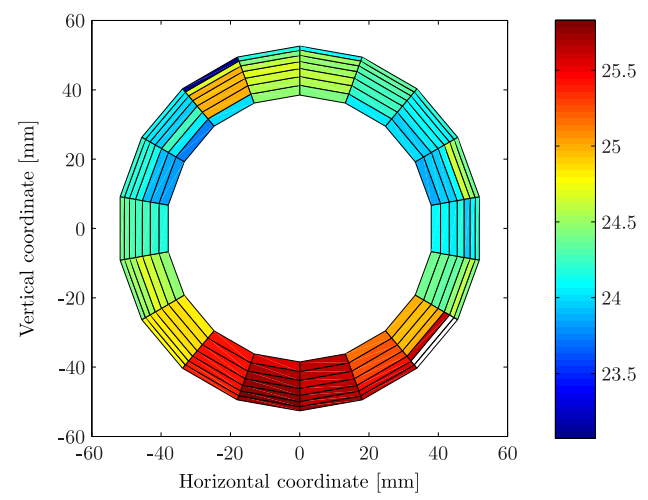

Figure 25: First natural frequency $\omega_{O 1}$ in $[\mathrm{Hz}]$ for a $\mathbf{A 2 0}$ phantom.

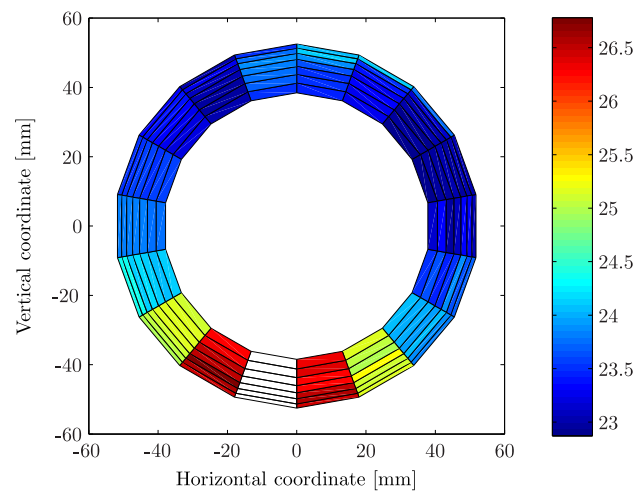

Figure 26: First natural frequency $\omega_{O 1}$ in $[\mathrm{Hz}]$ for a $\mathbf{B 2 0}$ phantom.

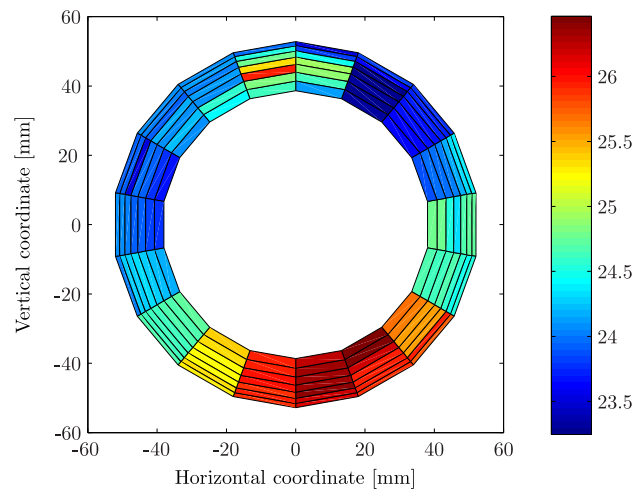

Figure 27: First natural frequency $\omega_{O 1}$ in $[\mathrm{Hz}]$ for a $\mathbf{C 2 0}$ phantom. 


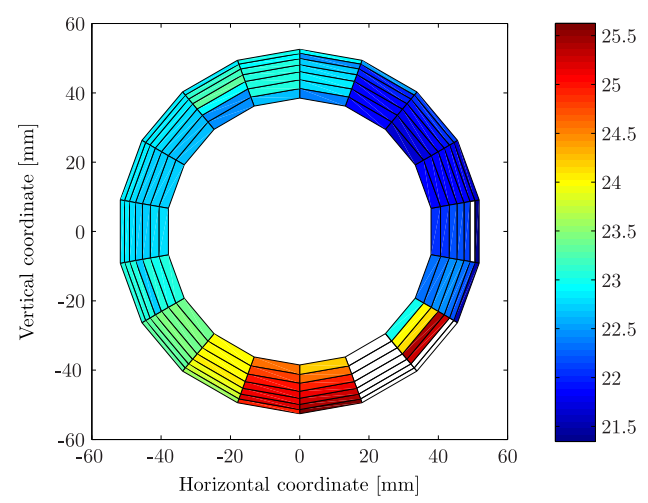

Figure 28: First natural frequency $\omega_{O 1}$ in $[\mathrm{Hz}]$ for a $\mathbf{D 2 0}$ phantom.

\section{B Analysis on the second natural frequency for all thirtheen phantoms}

The results of the analysis on the second natural frequency for all thirteen phantoms are shown below in Figures 29 41. Tumor size and position is given corresponding to Figure 2b. All figures on the left show the second natural frequency $\omega_{O 2}$ for the indicated phantom. The gaps in the colormap represent bad model fits due to noise and gaps in the data. The figures on the right show the result of the $t$-test. Here, red areas indicate rejection of the null hypothesis, presumably due to a stiffer inclusion. Blue areas indicate acceptance of the null hypothesis.

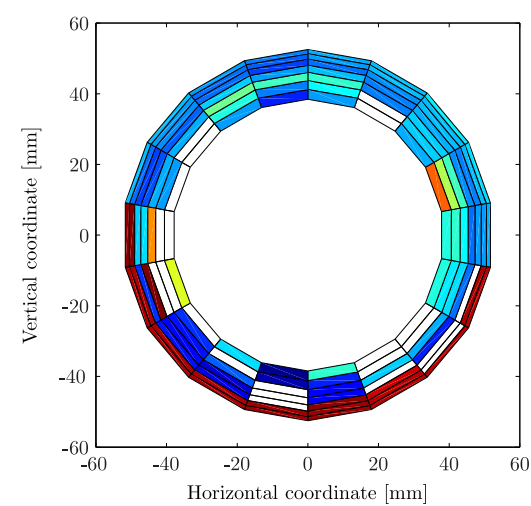

(a)
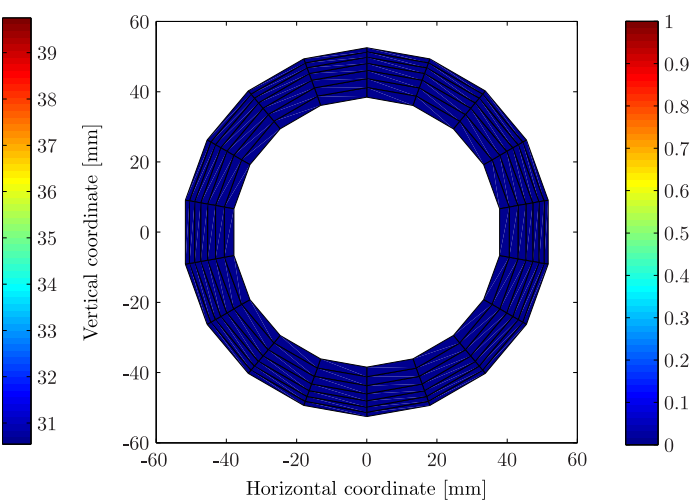

(b)

Figure 29: Results for a healthy phantom; (a) Second natural frequency $\omega_{\mathrm{O} 2}$ in [Hz], (b) result of the $t$-test where red indicates rejection and blue indicates acceptance of the null hypothesis. 


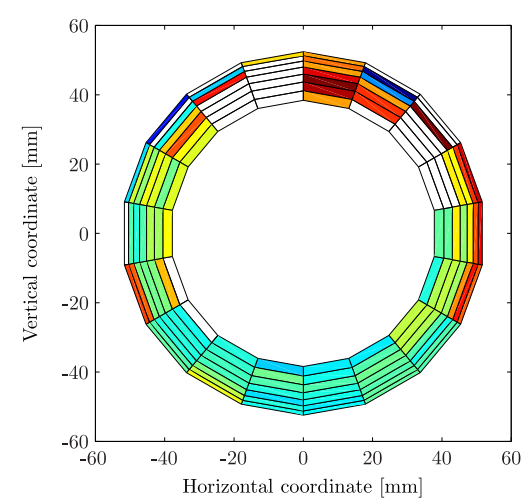

(a)
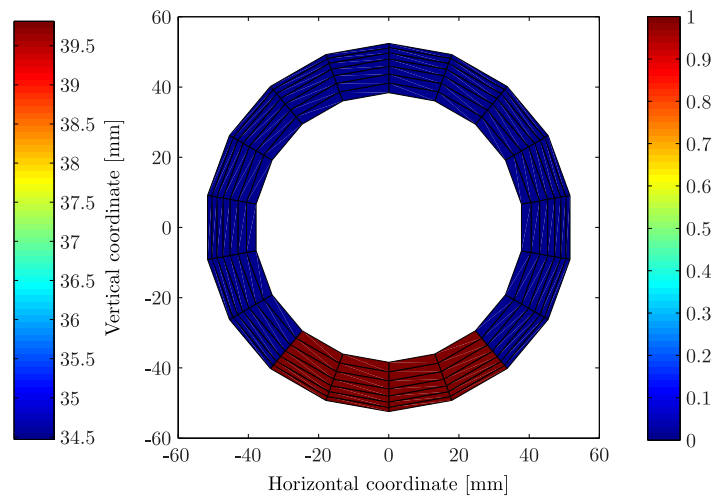

(b)

Figure 30: Results for a A5 phantom; (a) Second natural frequency $\omega_{O 2}$ in $[\mathrm{Hz}]$, (b) result of the $t$-test where red indicates rejection and blue indicates acceptance of the null hypothesis.

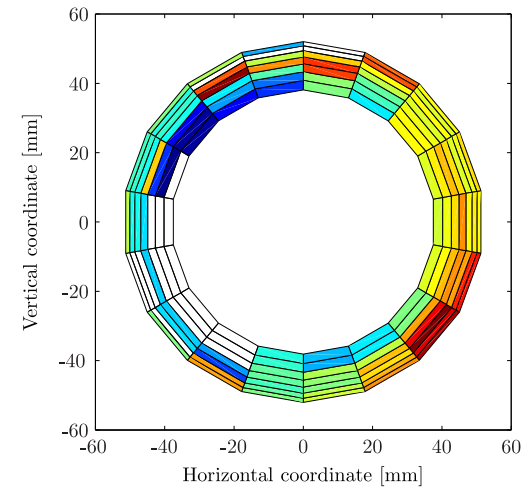

(a)
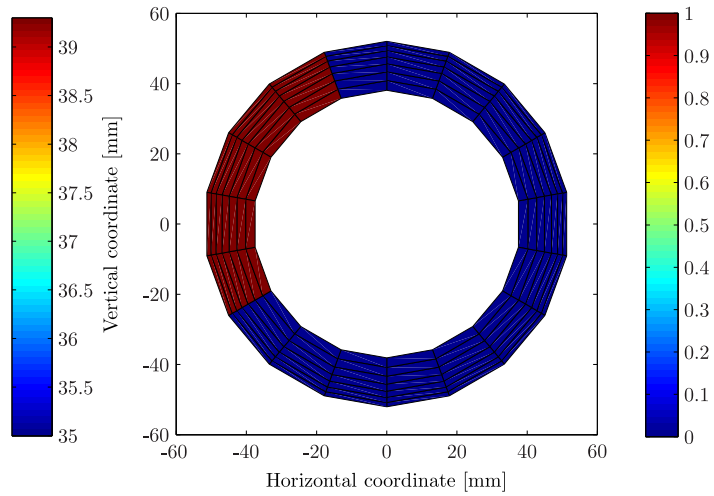

(b)

Figure 31: Results for a B5 phantom; (a) Second natural frequency $\omega_{O 2}$ in [Hz], (b) result of the $t$-test where red indicates rejection and blue indicates acceptance of the null hypothesis.

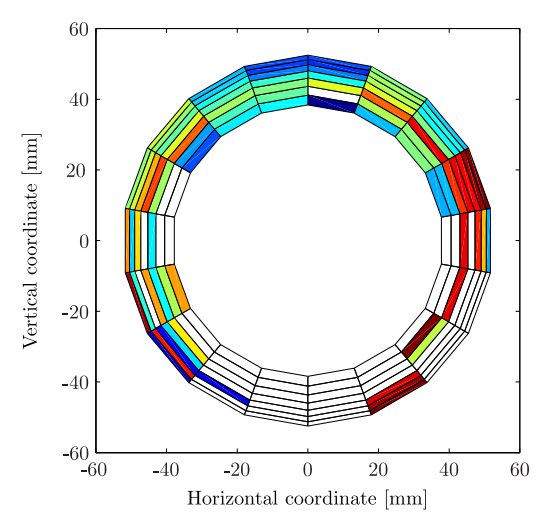

(a)
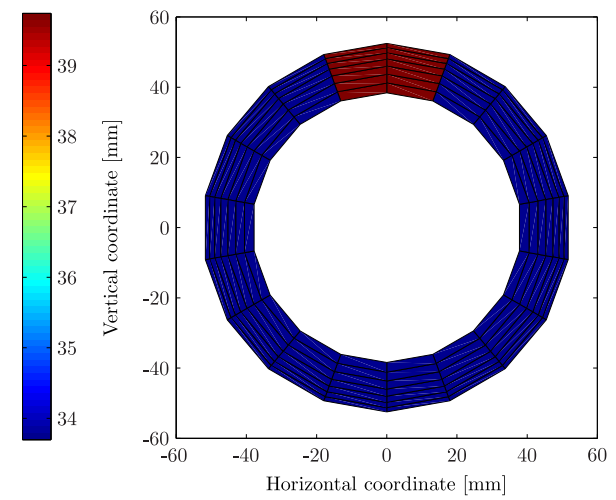

(b)

Figure 32: Results for a C5 phantom; (a) Second natural frequency $\omega_{O 2}$ in $[\mathrm{Hz}]$, (b) result of the $t$-test where red indicates rejection and blue indicates acceptance of the null hypothesis. 


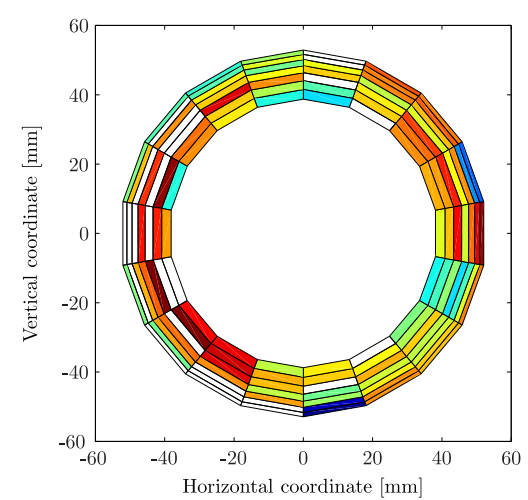

(a)
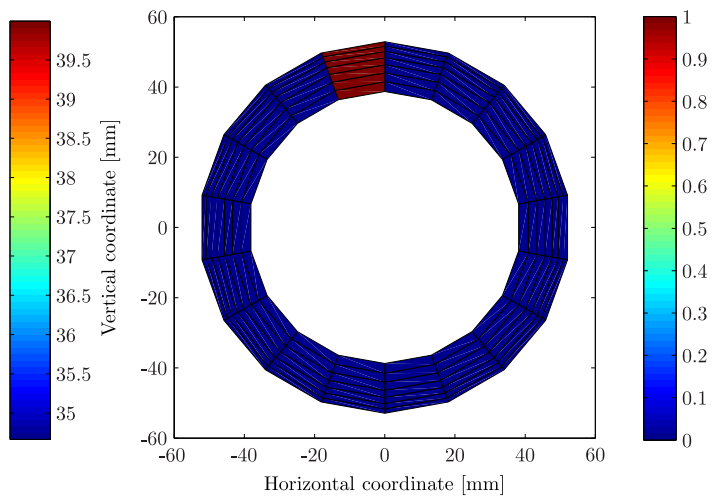

(b)

Figure 33: Results for a D5 phantom; (a) Second natural frequency $\omega_{O 2}$ in $[\mathrm{Hz}]$, (b) result of the $t$-test where red indicates rejection and blue indicates acceptance of the null hypothesis.

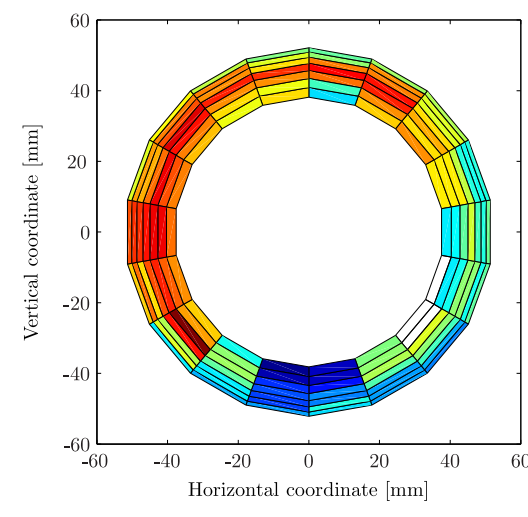

(a)
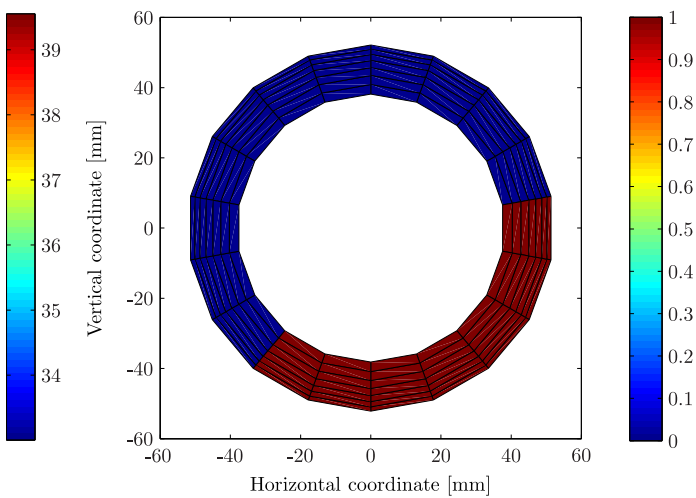

(b)

Figure 34: Results for a A10 phantom; (a) Second natural frequency $\omega_{\mathrm{O} 2}$ in $[\mathrm{Hz}]$, (b) result of the $t$-test where red indicates rejection and blue indicates acceptance of the null hypothesis.

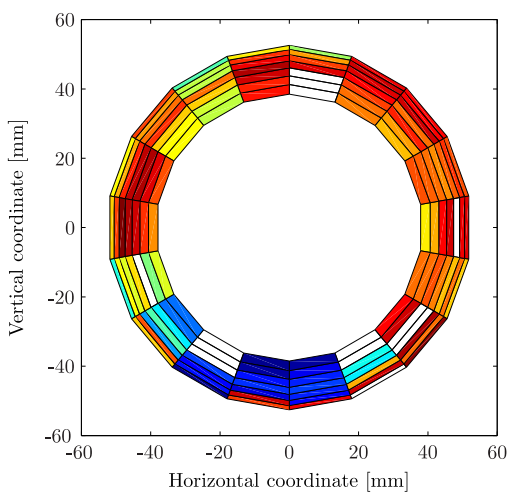

(a)
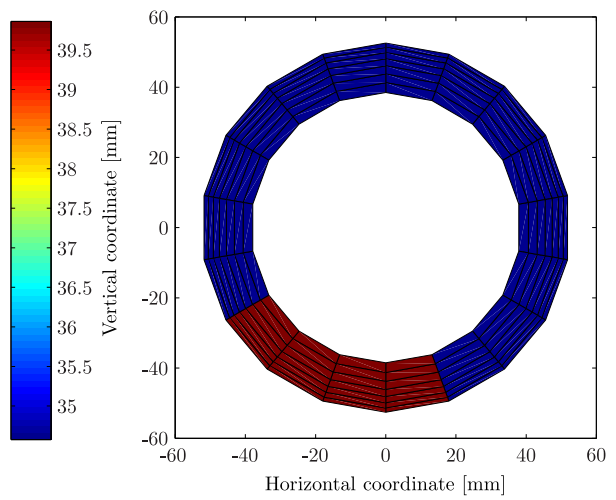

(b)

Figure 35: Results for a B10 phantom; (a) Second natural frequency $\omega_{\mathrm{O} 2}$ in $[\mathrm{Hz}]$, (b) result of the $t$-test where red indicates rejection and blue indicates acceptance of the null hypothesis. 


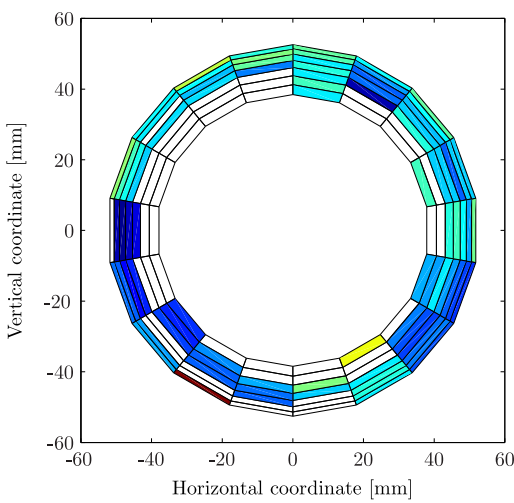

(a)
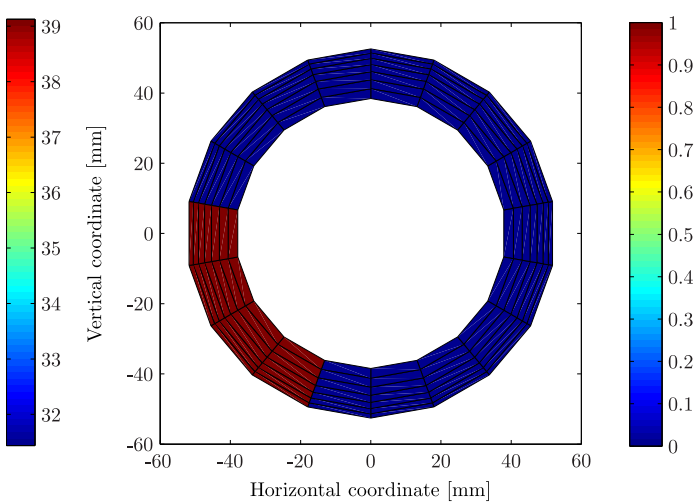

(b)

Figure 36: Results for a C10 phantom; (a) Second natural frequency $\omega_{O 2}$ in $[\mathrm{Hz}]$, (b) result of the $t$-test where red indicates rejection and blue indicates acceptance of the null hypothesis.

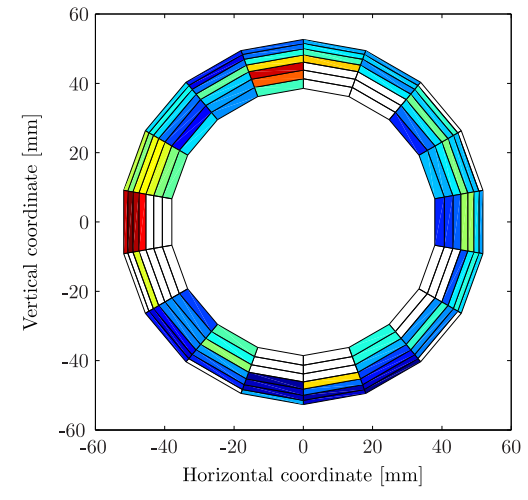

(a)
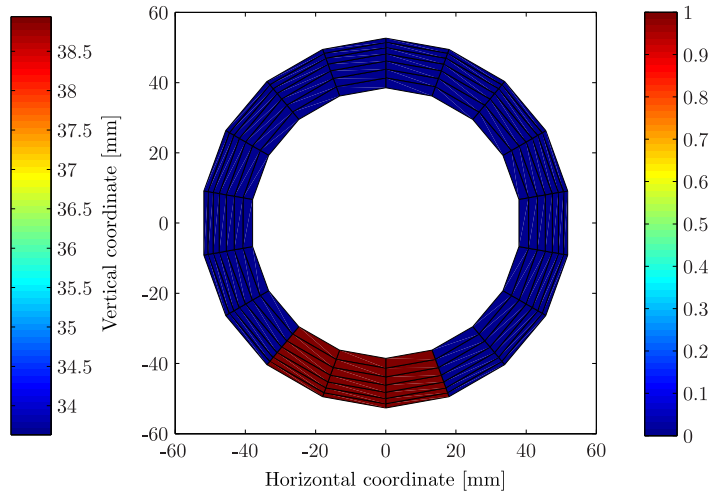

(b)

Figure 37: Results for a D10 phantom; (a) Second natural frequency $\omega_{\mathrm{O} 2}$ in $[\mathrm{Hz}]$, (b) result of the $t$-test where red indicates rejection and blue indicates acceptance of the null hypothesis.

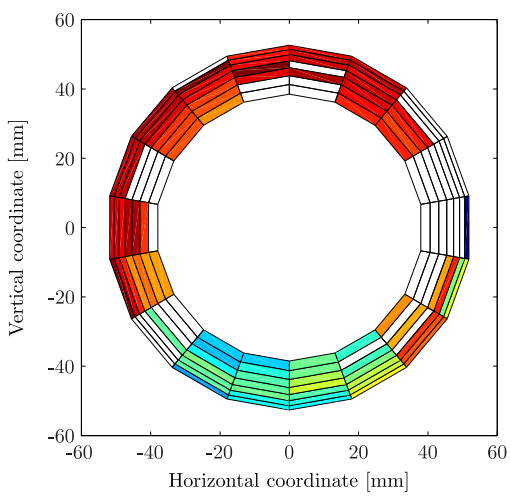

(a)
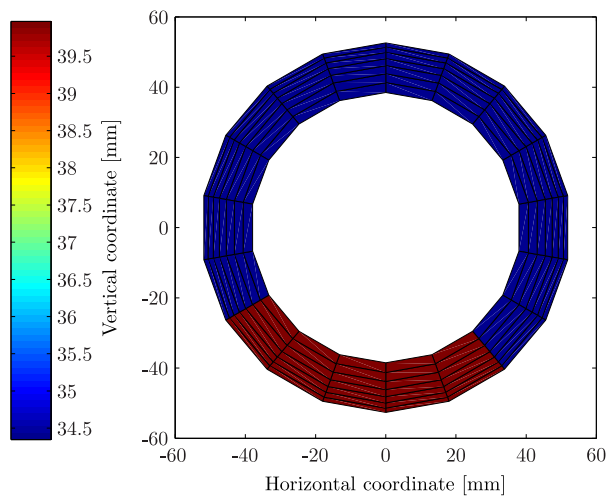

(b)

Figure 38: Results for a A20 phantom; (a) Second natural frequency $\omega_{\mathrm{O} 2}$ in $[\mathrm{Hz}]$, (b) result of the $t$-test where red indicates rejection and blue indicates acceptance of the null hypothesis. 


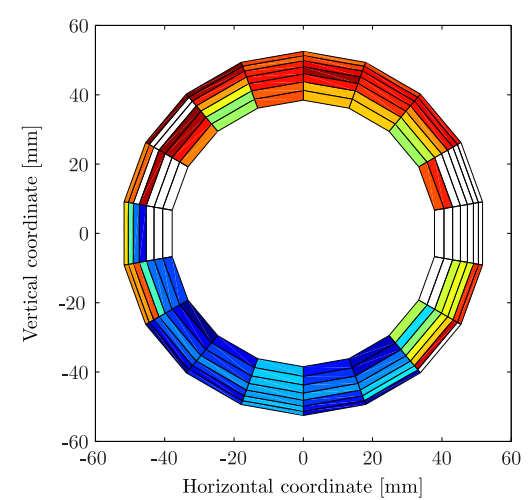

(a)
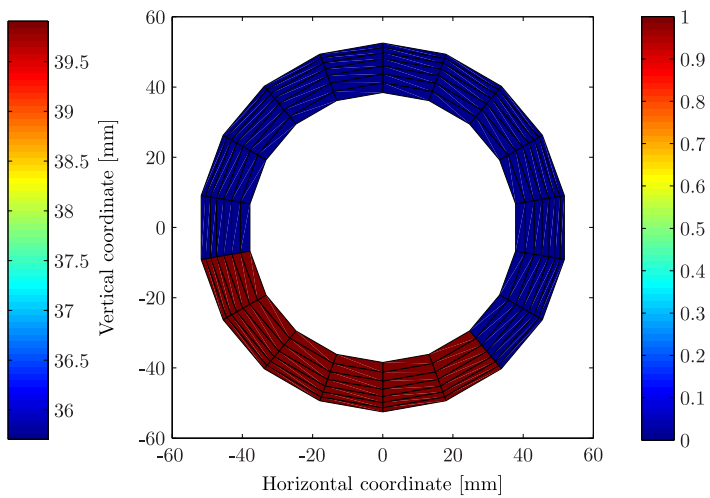

(b)

Figure 39: Results for a B20 phantom; (a) Second natural frequency $\omega_{\mathrm{O} 2}$ in $[\mathrm{Hz}]$, (b) result of the $t$-test where red indicates rejection and blue indicates acceptance of the null hypothesis.

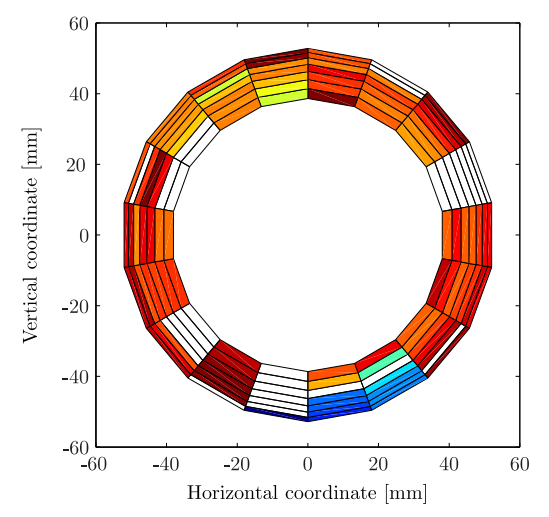

(a)
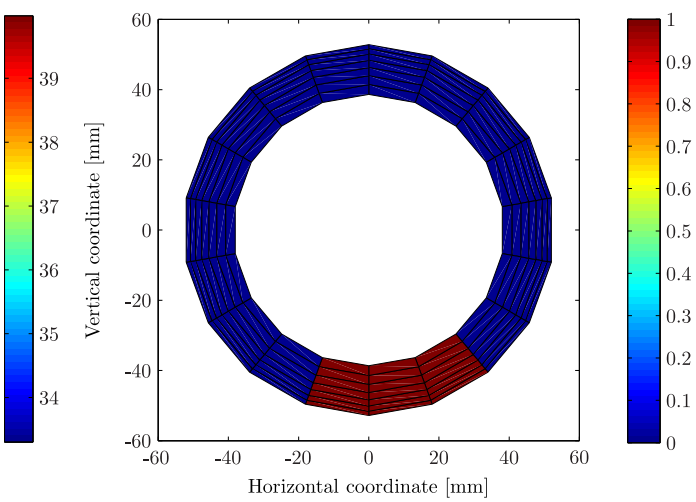

(b)

Figure 40: Results for a C20 phantom; (a) Second natural frequency $\omega_{\mathrm{O} 2}$ in $[\mathrm{Hz}]$, (b) result of the $t$-test where red indicates rejection and blue indicates acceptance of the null hypothesis.

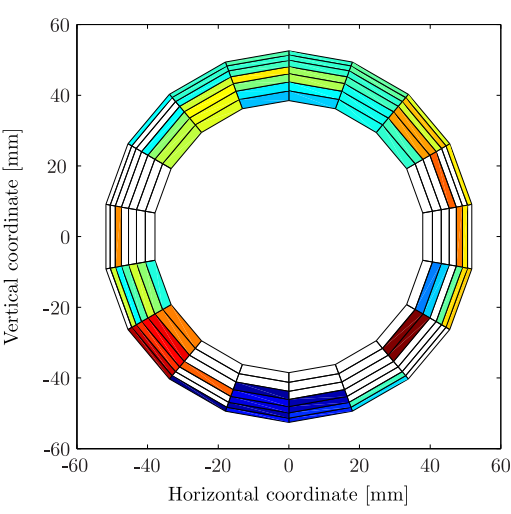

(a)
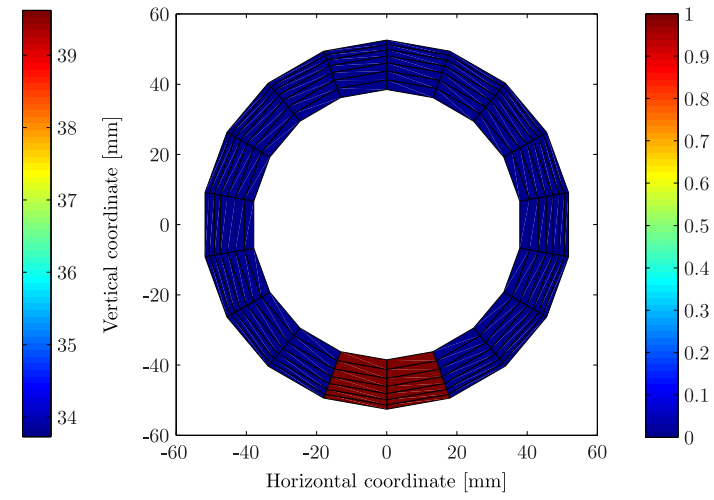

(b)

Figure 41: Results for a D20 phantom; (a) Second natural frequency $\omega_{\mathrm{O} 2}$ in $[\mathrm{Hz}]$, (b) result of the $t$-test where red indicates rejection and blue indicates acceptance of the null hypothesis. 